\title{
Error analysis for the lidar backward inversion algorithm
}

\author{
Francesc Rocadenbosch and Adolfo Comerón
}

\begin{abstract}
Here we depart from the inhomogeneous solution of a lidar equation using the backward inversion algorithm that is nowadays generally referred to as the Klett method. In particular, we develop an error sensitivity study that relates errors in the user-input parameters boundary extinction and exponential term in the extinction-to-backscatter relationship to errors in the inverted extinction profile. The validity of the analysis presented is limited only by the validity of application of the inversion algorithm itself, its numerical performance having been tested for optical depths in the $0.01-10$ range. Toward this end, we focus on an introductory background about how uncertainties in these two parameters can apply to a family of inverted extinction profiles rather than a single profile and on its range-dependent behavior as a function of the optical thickness of the lidar inversion range. Next, we performed a mathematical study to derive the error span of the inverted extinction profile that is due to uncertainties in the above-mentioned user calibration parameters. This takes the form of upper and lower range-dependent error bounds. Finally, appropriate inversion plots are presented as application examples of this study to a parameterized set of atmospheric scenes inverted from both synthesized elastic-backscatter lidar signals and a live signal. (C) 1999 Optical Society of America
\end{abstract}

OCIS codes: $\quad 010.0010,010.1290,010.3640$.

\section{Introduction}

Lidar inversion methods have been discussed for approximately three decades and are still under discussion. The method of using Bernoulli's differential equation form of the lidar equation (to be presented next) was proposed as early as 1954 by Hitschfeld and Bordan $^{1}$ to invert the rain rate from radar returns (although an unstable form) and has been applied or restated to invert lidar returns by, e.g., Barret and Ben-Dov, ${ }^{2}$ Viezee et al.,${ }^{3}$ Davis, ${ }^{4}$ Fernald et al..${ }^{5}$ Collis and Russell, ${ }^{6} \mathrm{Kohl},{ }^{7}$ and Klett, ${ }^{8}$ raising considerable controversy about its stability. Thanks to all these and other contributions, in 1981 Klett presented a stable analytical form for the inversion solution, ${ }^{8}$ and nowadays this method is generally referred to as the Klett method.

This is a straightforward nonadaptive inversion method that is used, for example, in automated ceilometers $^{9}$ and other lidar systems. Its most

The authors are with the Department of Signal Theory and Communications, Antennas, Microwaves, Radar, and Optics Group, Universitat Politecnica de Catalunya, C/Sor Eulalia de Anzizu s/n, 08034 Barcelona, Spain. The e-mail address for F. Rocadenbosch is roca@tsc.upc.es.

Received 13 October 1998; revised manuscript received 29 March 1999.

0003-6935/99/214461-14\$15.00/0

(C) 1999 Optical Society of America prominent feature is that of inverting inhomogeneous profiles (compared with the classic slope-method ${ }^{10}$ and least-squares fitting algorithms, ${ }^{11}$ which can invert only homogeneous atmospheres) at low computational cost and with excellent stability. Yet, in spite of the fact that the algorithm is nonadaptive (i.e., independent inversions are performed for each new incoming data burst), the reliability of the inverted profile is hampered by the accuracy of two user inputs to the algorithm (in its simplest form): the assumed boundary extinction at the far end of the inversion range and the assumed exponential term in the extinction-to-backscatter relationship.

Historically, authors who have studied different problems concerning the boundary condition are mainly Kunz, ${ }^{12}$ Ferguson and Stephans, ${ }^{13}$ Fernald, ${ }^{14}$ Sasano, ${ }^{15}$ Hughes et al., ${ }^{16}$ Klett, ${ }^{17}$ and Bissonnette. ${ }^{18}$ The assumption of different backscatter-to-extinction ratio forms in inversion algorithms has been discussed by Sasano and Nakane, ${ }^{19}$ Klett, ${ }^{20}$ and Keastner ${ }^{21}$ among others. Even with these relevant contributions and the sensitivity analysis of Bissonnette $^{18}$ (which was oriented to test the stability of different solution forms), there is still a void that encompasses the derivation of practical error bounds in the inverted extinction that are due to error bounds in the user calibrations. To fill this void, our goal is to derive different pairs of range-dependent error functions (referred to as upper and lower bounds) that limit the error span of the inverted ex- 
tinction in response to practical error margins for the above-mentioned user-input parameters of the algorithm.

\section{A. Review of the Klett Method}

Let us introduce the monostatic pulsed lidar equation. Under the assumption of single scattering, it takes the form

$$
P(R)=\frac{A}{R^{2}} \beta(R) \exp \left[-2 \int_{0}^{R} \alpha(r) \mathrm{d} r\right],
$$

where $P(R)$ represents the range-return power in watts, $\beta(R)$ is the range-dependent atmospheric backscatter coefficient of the atmosphere (in inverse kilometers times inverse steradians), $\alpha(R)$ is the range-dependent atmospheric extinction coefficient (in inverse kilometers), $R$ is the range (in kilometers), and $A$ is the system constant. Alternatively, Eq. (1) is often reformulated in differential form as

$$
\frac{\mathrm{d} S(R)}{\mathrm{d} R}=\frac{1}{\beta(R)} \frac{\mathrm{d} \beta(R)}{\mathrm{d} R}-2 \alpha(R),
$$

where $S(R)$ is the range-corrected power defined as

$$
S(R)=\ln \left[R^{2} P(R)\right] .
$$

It is obvious that the ultimate goal of lidar inversion is to retrieve both range-dependent extinction and backscatter functions, $\alpha(R)$ and $\beta(R)$, but, since this is an ambitious endeavor, different kinds of correlation hypotheses are introduced to simplify the problem. Thus, if the aerosol spectral form or composition does vary with location, $\beta(R)$ and $\alpha(R)$ can be regarded as having a proportionality that is range dependent so that the traditional way to relate both functions is to assume a power-law relationship of the form

$$
\beta(R)=B \alpha(R)^{k},
$$

where, in the most general case, both $B$ and $k$ are functions of $R$.

Here, we encounter different approaches: A most general one assumes that $k=1$ and a variable backscatter-to-extinction ratio of $B(R){ }^{20}$ As a further refinement, one can even distinguish between aerosol and molecular backscatter-to-extinction ratios, $B_{a}(R)$ and $B_{m}$, respectively. ${ }^{14,20}$ Even though a theoretical analysis of the errors caused by use of a constant backscatter-to-extinction ratio instead of a spatially variable ratio is discussed in Ref. 22 , under most circumstances it is not possible to know a priori the backscatter-to-extinction ratio as a function of the range and a constant ratio $B$ with range is used. This hypothesis has only a physical meaning if gaseous absorption is negligible (typical figures of the optical parameters lie in the ranges $\alpha=5 \times 10^{-2} 55$ $\mathrm{km}^{-1}, \beta=3.5 \times 10^{-4}$ to $2.7 \mathrm{~km}^{-1} \mathrm{sr}^{-1}$ ) and there are no multiple-scattering effects. ${ }^{19,20}$ As a result of multiple scattering, the return power exceeds the value predicted by Eq. (1) because it includes photons that have been scattered more than once. In a practical application, multiple scattering can be neglected in visually clear (i.e., noncloudy) atmospheres in which the one-way optical thickness is less than approximately 1 , given the field of view of the receiving optics of several milliradians or less. These effects tend to be much more important in fog, cloud, and rain situations ${ }^{6}$ because of the much larger probability of scattering and the larger scattering particles, which focus a much larger fraction of scattered photons in near-forward directions.

Equivalently, the assumption of a constant ratio $B$ essentially assumes that the size distribution and composition of the aerosol scatterers do not change with range from the lidar and that variation in backscatter from aerosols is due to changes in their number density. ${ }^{14}$ This is the only hypothesis we can use without a priori information, and it can be justified only from the point of view of the numerical analysis of lidar data as a first inversion trial. The customary alternative approach, whereby information on backscatter and extinction is cast in the form of a power-law relationship of Eq. (4) with $B$ and $k$ as a pair of constants, has a less direct physical justification but nevertheless also has a practical value. ${ }^{20}$ Collis and Russell, ${ }^{6}$ Pinnick et al., ${ }^{23}$ and Russell et al. ${ }^{24}$ have provided values of the backscatter-toextinction ratio, which depends on the size distribution and complex refractive index of the aerosols and usually varies from 10 to $100 . .^{25,26}$ Reported values of $k$ are usually in the $0.67<k<1.3$ range. ${ }^{20,27,28}$

All things considered, the simple power-law approach of Eq. (4) with constants $B$ and $k$ has been shown by experiment to be reasonably $\operatorname{good}^{8}$ even though the transformation between $\alpha$ and $\beta$ is in general a many-to-one problem, as more than one value of $B$ can occur, at different locations, for the same value of $\alpha$ (see Ref. 20). See Refs. 27 and 29 for a deeper insight into the nonuniqueness problem of the backscatter-extinction relation.

Under these considerations, if Eq. (4) is substituted into Eq. (2) with constants $B$ and $k$, it becomes

$$
\frac{\mathrm{d} S(R)}{\mathrm{d} R}=\frac{k}{\alpha(R)} \frac{\mathrm{d} \alpha(R)}{\mathrm{d} R}-2 \alpha(R) .
$$

Note that the backscatter-to-extinction constant ratio $B$ is irrelevant for inversion of the extinction profile (but not the backscatter profile) and vanishes [see also Eq. (7)]. Only $k$ is retained as a reminder of the correlation between extinction and backscatter.

Equation (5) is a Bernouilli differential equation in $\alpha(R)$ and, when a calibration of the extinction coefficient at the maximum inversion range $R_{m}$ is fed to the algorithm ${ }^{6,30}$ as

$$
S\left(R_{m}\right)=S_{m} \leftrightarrow \alpha\left(R_{m}\right)=\alpha_{m},
$$


the backward solution proposed by Klett arises:

$$
\alpha(R)=\frac{\exp \left[\left(S-S_{m}\right) / k\right]}{\alpha_{m}{ }^{-1}+\frac{2}{k} \int_{R}^{R_{m}} \exp \left[\left(S-S_{m}\right) / k\right] \mathrm{d} r} .
$$

This solution, with which the extinction coefficient is calibrated at the maximum range $R_{m}$, is highly stable because, as $R$ decreases from the end of the exploring range, where the signal-to-noise ratio is lower, $\alpha(R)$ in Eq. (7) becomes the ratio of two numbers progressively larger. [Here, it is assumed that $S(R)$ generally decreases with increasing range $R$. This occurs in most practical applications because of the effect of the extinction term in Eq. (1).]

Following this simplified approach, a refinement of practical advantage is to divide the atmosphere into layers as in the so-called slice method of inversion, which is merely an extremely close variant of the slope method but applied to successive range intervals. ${ }^{31}$ In its application to the Klett method, $B$ and $k$ are allowed to vary among the layers. Throughout this study we consider the oversimplification of having one single slice in the inversion interval (sometimes, even in front of substantial variations in the return signal) in order to test the robustness of the error bounds that are derived here.

\section{B. Calibration Problem Facts}

Except when balloonborne and plenty of cooperative equipment is used, selection of $k$ and the boundary extinction $\alpha_{m}$ is always a problem. For optical depths greater than unity, an adequate estimate of $\alpha_{m}$ can usually be obtained with the well-known slope method as minus one half of the average slope of the $S(R)$ curve $^{30}$ [the assumption of a homogeneous atmosphere in the case of the slope method is equivalent to $\mathrm{d} \alpha / \mathrm{d} R=0$ in Eq. (5) and, hence, $\alpha_{m} \approx 1 / 2$ $S(R)$ ]. Of course, this approach often becomes quite inaccurate and is risky for less turbid atmospheres because microstructure variation along the lidar beam path could easily lead to relatively large fluctuations of the fractional gradient of attenuation $k / \alpha \mathrm{d} \alpha / \mathrm{d} R$ compared with $-2 \alpha(R)$ in Eq. (5), hence invalidating local application of the slope method. Although the utility of the slope method increases with increased optical depth, ${ }^{8}$ it is also necessary that a priori information confirm (1) the existence of a homogeneous atmosphere at the last slice of the inversion interval used to calibrate the boundary extinction and (2) enough signal-to-noise ratio. If this information is unavailable point (1) could yield to situations in which an inhomogeneous reflection (e.g., a cloud reflection) could not be distinguished from the returns of a homogeneous atmosphere (see Appendix B in Ref. 29), and point (2) could yield to situations in which the return signal would be buried in noise.

Similar comments apply but with higher noise immunity when the selected algorithm to estimate the boundary extinction over the interval $\left(R, R_{m}\right)$ is a curve-fitting exponential ${ }^{11}$

$$
\begin{aligned}
\min _{a, b}\left\{\left\|R^{2} P(R)-b \exp (-a R)\right\|^{2}\right\} & \\
& =\min _{a, b}\left\{\sum_{i=1}^{N}\left[R_{i}{ }^{2} P\left(R_{i}\right)-b \exp \left(-a R_{i}\right)\right]^{2}\right\},
\end{aligned}
$$

instead of the classic slope method. In Eq. (8) the norm minimization is performed over the variables $a=2 \alpha$ and $b=A \beta$ for all the points $R_{i} \in\left(R, R_{m}\right)$. Hence, an estimate $\alpha_{c}$ of the true calibration $\alpha_{m}$ is obtained as $\alpha_{c}=a / 2$.

Finally, another uncertainty example ${ }^{30}$ is that, if one imposes the constraint that the average extinction over $\left[0, R_{m}\right]$ is the same as over the last slice $\left[R_{0}, R_{m}\right]$, both high and low visibility boundary values of extinction can be found for a given return signal, and so the question arises as to how one can discriminate between the two possibilities. Again, the answer must come from appropriate $a$ priori information such as direct observation (under many circumstances) or cooperative instrumentation.

As for the correlation constant $k$ of Eq. (4), even an estimate is difficult to achieve and, in practice, this value comes from a few values reported in the literature. ${ }^{27,28}$

In short, $k$ and $\alpha_{m}$ represent properties of the atmospheric aerosols that are impossible to calibrate perfectly, which serves to justify that the core of this paper is to study the sensitivity of the inverted extinction to $k$ and $\alpha_{m}$.

\section{Limits of Validity of the Study}

The error study tends to be as general as possible, its validity being limited only insofar as application of the Klett method by use of the power-law relationship of Eq. (4) and a constant value for $B$. Thus, we conducted and tested the study from a theoretical point of view to ensure an optical thickness application range as wide as $\tau \approx 0.01-10$ [optical thickness definitions are discussed later in Eqs. (13)-(14)]. We realize that this could encompass situations in which molecular scattering or multiple scattering effects (depending on the field of view of the receiving optics) would become significant and, therefore, hamper strict application of the inversion method. Nevertheless, in this case, since the multiple-scattering equation basically reduces the conventional singlescattering lidar equation using correction terms, ${ }^{32}$ the following analysis can still be extrapolated by the potential user. Weinman, ${ }^{33}$ for example, gives a single-scattering inversion solution for the lidar return measured by a central field-of-view detector at which a linear backscatter-to-extinction relation is assumed. ${ }^{32}$

\section{Inversion Problem as a Family of Functions}

With the practical uncertainties in the estimation of the input parameters of Eq. (7), $k$ and $\alpha_{m}$, the inverted profile $\hat{\alpha}\left(R, k_{c}, \alpha_{c}\right)$ from user-selected values of $k_{c}$ and $\alpha_{c}$ become a single representative of the two- 
dimensional family yielded by Eq. (2) when the selected pairs $\left(k_{c}, \alpha_{c}\right)$ sweep the user error span:

$$
\begin{gathered}
k_{c, \text { min }}<k_{c}<k_{c, \text { max }}, \\
\alpha_{c, \text { min }}<\alpha_{c}<\alpha_{c, \text { max }} .
\end{gathered}
$$

Subscripts max and min indicate maximum and minimum limits of the selected values for these parameters, and we assume that they were chosen conservatively so that they always include the true atmospheric pair, $k$ and $\alpha_{m}$ (unknown by the user).

The relationship between the user-inverted profile $\hat{\alpha}\left(R, k_{c}, \alpha_{c}\right)$ and the true atmospheric extinction profile $\alpha\left(R, k, \alpha_{m}\right)$ in Eq. (7) is derived in the Appendix and takes the form

$$
\hat{\alpha}\left(R, k_{c}, \alpha_{c}\right)=\frac{\alpha(R)^{k / k_{c}} I\left(R, k_{c}, \boldsymbol{\alpha}\right)}{\frac{\alpha_{m}{ }^{k / k_{c}}}{\alpha_{c}} I\left(R_{m}, k_{c}, \boldsymbol{\alpha}\right)+\frac{2}{k_{c}} F\left(R, k, k_{c}, \boldsymbol{\alpha}\right)},
$$

where $I\left(R, k_{c}, \boldsymbol{\alpha}\right)$ and $F\left(R, k, k_{c}, \boldsymbol{\alpha}\right)$ are auxiliary functions defined as

$$
\begin{gathered}
I\left(R, k_{c}, \boldsymbol{\alpha}\right)=\exp \left[-\frac{2}{k_{c}} \int_{R_{0}}^{R} \alpha(r) \mathrm{d} r\right], \\
F\left(R, k, k_{c}, \boldsymbol{\alpha}\right)=\int_{R}^{R_{m}} \alpha(r)^{k / k_{c}} I\left(r, k_{c}, \boldsymbol{\alpha}\right) \mathrm{d} r .
\end{gathered}
$$

The cap over $\alpha$ means estimate of, $\alpha$ is a reminder of the functional dependency of any function on the atmospheric profile $\alpha(R)$, and $R_{0}$ is some predetermined minimum range that becomes irrelevant in Eq. (10). Equation (10) describes the inverted extinction profile in terms of the atmospheric profile $\alpha(R)$, the atmospheric pair $\left(k, \alpha_{m}\right)$, and the user-selected pair $\left(k_{c}\right.$, $\alpha_{c}$ ). Equation (10) is of advantage only for simulation and study purposes because it relates the true atmospheric extinction profile (e.g., a computersynthesized profile) with parameters $\left(k, \alpha_{m}\right)$ to the user-inverted extinction profile with parameters $\left(k_{c}\right.$, $\alpha_{c}$ ) without the need to compute the logarithmic range-corrected power $S(R)$ as in Eq. (7). Obviously, $\hat{\alpha}\left(R, k_{c}, \alpha_{c}\right) \rightarrow \alpha(R)$ as long as $\left(k_{c}, \alpha_{c}\right) \rightarrow\left(k, \alpha_{m}\right)$.

Figure 1 illustrates an example of the problem by comparison of a synthesized atmospheric extinction profile $\alpha\left(R, k, \alpha_{m}\right)$ to a set of user-inverted profiles $\hat{\alpha}\left(R, k_{c}, \alpha_{c}\right)$. The atmospheric profile, which is labeled as true extinction, is much the same as the one used by Klett to test the stability of his algorithm in Ref. 8. It was built so that the extinction of the bottom base of the trapezium platform is $\alpha$ and that of the top base is $2 \alpha$. In practice, this could be representative of an exploration path where inhomogeneities are not very large (a factor of 2 in the simulations).

Before proceeding further, we find it convenient to describe the atmospheric condition in terms of the
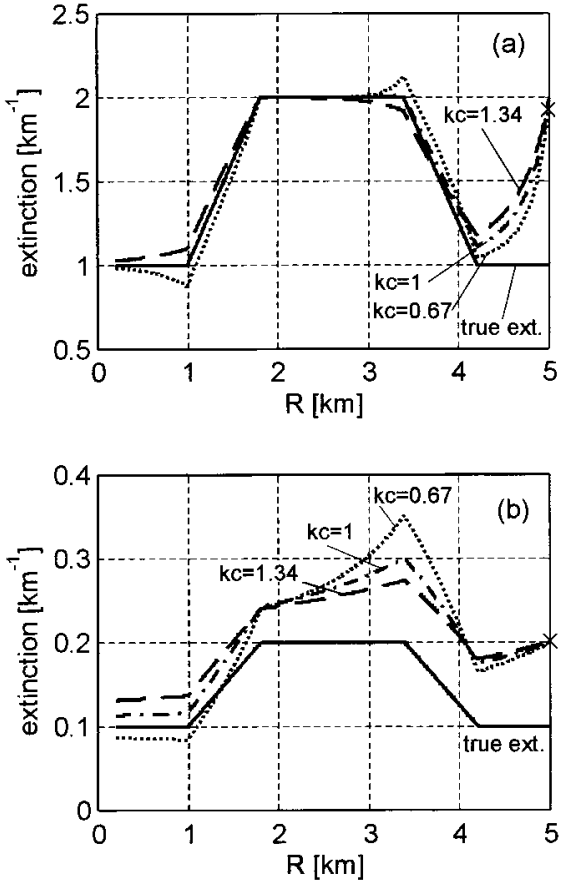

Fig. 1. Trapezium profile. Solutions of the synthesized lidar signals according to Eq. (10) with a common shape of the extinction profile (true ext.) by use of different values for $k_{c}\left(0.67 \leq k_{c} \leq 1.34\right)$ and the boundary condition $\alpha_{c}=2 \alpha_{m}$. The $\times$ indicates the userselected boundary extinction $\alpha_{c}$ at $R_{m}$. Simulations are parameterized for optical thicknesses of (a) $\tau=7.4$ and (b) $\tau=0.74$.

optical thickness or path-integrated extinction coefficient

$$
\tau\left(R_{\min }, R_{\max }\right)=\int_{R_{\min }}^{R_{\max }} \alpha(r) \mathrm{d} r
$$

or, equivalently, as the two-way transmission

$$
T=10 \log [\exp (-2 \tau]=-8.686 \tau(\mathrm{dB})
$$

rather than use of the terms bottom base and top base extinction.

In addition, it is also necessary to define the words family and branch within the context of Fig. 1 and the like. We define a family as a bundle of plots that leave from the same user-selected boundary extinction $\alpha_{c}$ at $R_{m}$ and a branch as any curve that belongs to a family. Each branch of a family is defined by a user-selected $k_{c}$.

Using the above definitions, we plotted a family of curves in Fig. 1, all of which are solutions of the lidar equation by use of Klett's method with different boundary conditions $\left(k_{c}, \alpha_{c}\right)$ in Eq. (7), in response to synthesized lidar signals generated from a common shape in the extinction profile. Figure 1(a) corresponds to an optical thickness $\tau=7.4(T=-64.3 \mathrm{~dB})$, whereas Fig. 1(b) corresponds to $\tau=0.74(T=-6.5$ $\mathrm{dB})$. In both cases, the two families shown are miscalibrated up to $\alpha_{c}=2 \alpha_{m}$ and each branch $\hat{\alpha}\left(R, k_{c}, \alpha_{c}\right)$ corresponds to curves with $k_{c}=0.67,1$, and 1.34 . Each family has been computed according to Eq. (10) 

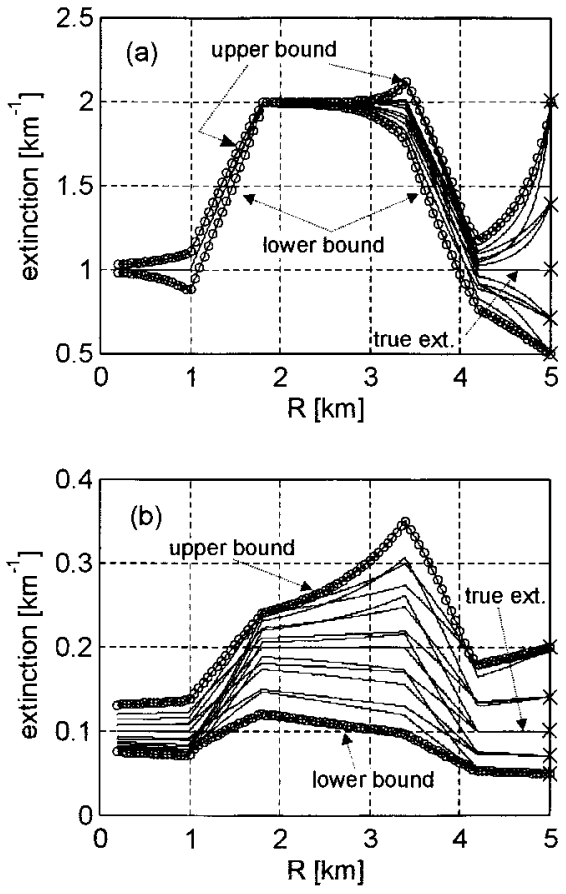

Fig. 2. Trapezium profile. Same as Fig. 1 but $k_{c}$ and $\alpha_{c}$ span the user-error margin $\alpha_{m} / 2 \leq \alpha_{c} \leq 2 \alpha_{m}$ and $0.67 \leq k_{c} \leq 1.34$ [expression (9)].

in response to the synthesized atmospheric test profile $\alpha\left(R, k, \alpha_{m}\right)$ for which $k=1$ and $\alpha_{m}=1 \mathrm{~km}^{-1}$.

At first glance, one might wonder whether the example of Fig. 1(a) is at all representative considering that such an optical thickness $(\tau=7.4)$ plus the geometric attenuation $R^{2}$ would yield to a low signal-tonoise ratio at $R_{m}$. However, it must be said that the same plots are reencountered when abscissas are downscaled by some predefined factor and, consequently, when the optical thickness has more practical values.

To study the sensitivity of the inverted extinction profile to values of the pair $\left(k_{c}, \alpha_{c}\right)$, in Fig. 2 we plotted ten profiles $\hat{\alpha}\left(R, k_{c}, \alpha_{c}\right)$ inverted in response to the same input test profiles as in Fig. 1 (this profile is labeled as true ext.) when $k_{c}$ and $\alpha_{c}$ sweep the range $k_{c, \text { min }}=0.67$ and $k_{c, \text { max }}=1.34$ (in three linear steps) and $\alpha_{c, \min }=\alpha_{m} / 2$ and $\alpha_{c, \max }=2 \alpha_{m}$ (in five log steps) according to expression (9). The latter error interval represents a factor of 2 misestimation from $\alpha_{m}$ in the user-selected boundary extinction $\alpha_{c}$. Superimposed on each plot, Fig. 2 depicts the sought-after upper and lower error bounds of the inverted extinction. These bounds are the best in the sense that they represent the envelope of the two-dimensional function $\hat{\alpha}\left(R, k_{c}, \alpha_{c}\right)$ when its principal variables $\left(k_{c}\right.$, $\alpha_{c}$ ) are within the user-uncertainty span of expression (9). Based on the graphic results obtained, we determined that computation of these uncertainty or error bounds becomes more complicated because branches that belong to different families intersperse and overlap along the inversion range. As a result, the upper and lower envelope representatives of the uncertainty interval in the inverted extinction, which we want to determine, are formed by a myriad of branches instead of by two particular ones.

We tackle this problem in subsequent sections.

In the examples of Figs. 1 and 2, where the atmospheric profile is simulated and hence is perfectly known, reconstruction errors in the solution can be calculated from Eq. (10) as

$$
\epsilon\left(R, k_{c}, \alpha_{c}, k, \alpha_{m}\right)=\left[1-\frac{\hat{\alpha}\left(R, k_{c}, \alpha_{c}\right)}{\alpha\left(R, k, \alpha_{m}\right)}\right] 100 \% .
$$

Error $\epsilon$ is a range-dependent function and it depends on the particular atmospheric extinction profile at hand, the boundary range $R_{m}$, the assumed pair $\left(k_{c}\right.$, $\left.\alpha_{c}\right)$, and the atmospheric pair $\left(k, \alpha_{m}\right)$.

A comprehensive set of simulations has been performed to help to reveal significant trends about the sensitivity of the inverted extinction to the pair $\left(k_{c}\right.$, $\alpha_{c}$ ) for different optical depths. We can conclude that:

(1) The solution is less sensitive to the boundary value ratio for increasing optical depths [this can also be inferred from comparison of Fig. 2(a) with Fig. 2(b)]. An explanation for this is that, for increased optical depths, $\alpha_{c}$ becomes a large number. Its inverse in Eq. (7) is a small number and, hence, the backward solution simply becomes the ratio of the normalized signal and the path-integrated normalized signal. Consequently, the influence of the remote calibration vanishes. This coincides with the criterion $^{8}$ that, for $\tau>1$, the inversion method can be applied in principle by using only that information contained in the signal itself. In other words, it is a question of the range of optical thickness that determines the convergence to the true extinction.

(2) Overestimates of the boundary value are preferred to underestimates, (which is shown analytically in Eq. (A6) under the simplification that $k_{c}=k$.

(3) In homogeneous intervals, the choice of $k_{c}$ is completely irrelevant because the term $k / \alpha \mathrm{d} \alpha / \mathrm{d} R$ in Eq. (2) tends to zero and the inverted profiles tend to be parallel lines in these intervals [consider, for example, Fig. 2(b) between the 1.8- and 3.39-km range interval]. For the same reason, in inhomogeneous intervals for which heterogeneities are not very large (approximately a factor of 2), the sensitivity of the inverted extinction profile to errors in $k_{c}$ is usually outweighed by errors in the boundary extinction $\alpha_{c}$.

(4) $\epsilon(R)$ is generally a nonmonotonic decreasing function with increasing distance from the boundary calibration at $R_{m}$. Precise conclusions about the variation of the value $\epsilon$ with range can hardly be derived because errors depend on the aerosol distribution. Thus, in Fig. 2(a) $\epsilon(R)$ increases in the range between 0.5 and $1.5 \mathrm{~km}$, even though there is an overall decreasing trend as one moves backward from the boundary [see also Fig. 2(b)].

The conclusions outlined here from the simulation results presented are in close agreement with those of 


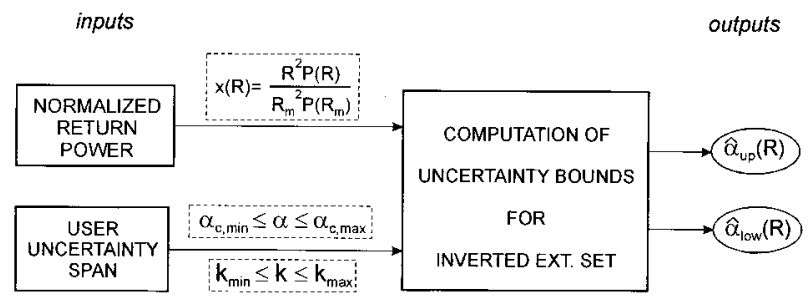

Fig. 3. Flow diagram indicating inputs and outputs of the error bound computation algorithms for the inverted extinction given $a$ priori uncertainty information.

Sasano and Nakane. ${ }^{19}$ In a practical inversion, however, Eq. (7) would become more sensitive to the measurement noise and this would also contribute to an increase in inversion errors.

\section{Some Absolute Bounds}

In Section 2 we showed that a two-dimensional set of solutions exists for one lidar signal if no a priori information is available. Otherwise, the useruncertainty intervals described by expression (9) can be translated into appropriate error bounds for the inverted extinction of the form

$$
\alpha_{\text {low }}(R) \leq \hat{\alpha}\left(R, k_{c}, \alpha_{c}\right) \leq \alpha_{\text {up }}(R),
$$

where $\alpha_{\text {up }}(R)$ and $\alpha_{\text {low }}(R)$ correspond to the upper and lower error bounds, respectively. Here we are committed to the analytical derivation of absolute bounds for lidar return signals $P(R)$, even though these bounds are not the closest and, obviously, not the most unique for all range $R$, but they are a first approximation to them. Inputs and outputs of the computation procedure are sketched in Fig. 3.

The key to the mathematical procedure lies in rewriting Eq. (7) in terms of an auxiliary function [see also Eq. (3)],

$$
x(R)=\exp \left[S(R)-S_{m}\right]=\frac{R^{2} P(R)}{R_{m}{ }^{2} P\left(R_{m}\right)},
$$

and of the auxiliary variables,

$$
\gamma=1 / k_{c} ; \quad a=1 / \alpha_{c},
$$

so that Eq. (7) becomes

$$
\hat{\alpha}(R, \gamma, a)=\frac{x(R)^{\gamma}}{a+2 \gamma \int_{R}^{R_{m}} x(r)^{\gamma} \mathrm{d} r} .
$$

In the future, the pairs $(\gamma, a)$ and $\left(k_{c}, a_{c}\right)$ will be used indiscriminately. Note that to this end Eq. (19) remains the backward inversion method similar to Eq. (7), given that $\gamma$ and $\alpha$ are now the user-selected parameters.

By using the basic properties of $x^{\gamma}$ and that of $x(R)$ $>0$, one can easily limit this function between two others of the same kind, for example,

$$
x(R)^{\gamma_{\min }}<x(R)^{\gamma}<x(R)^{\gamma_{\max }} \text { for } x(R)>1,
$$

$$
x(R)^{\gamma_{\max }}<x(R)^{\gamma}<x(R)^{\gamma_{\min }} \text { for } x(R)<1 .
$$

These inequalities turn into equalities for $x(R)=1$ because all the curves of the family coincide at the point $(1,1) . \quad \gamma_{\min }$ and $\gamma_{\max }$ were computed from the uncertainty interval of $k_{c}$ with expression (9).

By introducing the definitions of the Heaviside and delta functions, denoted $u(x)$ and $\delta(x)$, respectively,

$$
\begin{aligned}
& u(x) \equiv\left\{\begin{array}{ll}
1 & x \geq 1 \\
0 & \text { otherwise }
\end{array},\right. \\
& \delta(x) \equiv\left\{\begin{array}{ll}
1 & x=0 \\
0 & \text { otherwise }
\end{array},\right.
\end{aligned}
$$

We expressed inequalities (20) in terms of the upper and lower bounds of $x(R)^{\gamma}, y_{\text {up }}(R)$, and $y_{\text {low }}(R)$, respectively, as follows:

$$
\begin{aligned}
& y_{\text {up }}(R)=x^{\gamma_{\max }} u(x-1)+x^{\gamma_{\min }} u(1-x)-\delta(x-1), \\
& y_{\text {low }}(R)=x^{\gamma_{\min }} u(x-1)+x^{\gamma_{\max }} u(1-x)-\delta(x-1),
\end{aligned}
$$

where variable $R$ is a reminder of the range dependence of $x(R)$.

We achieved the derivation of the absolute rangedependent bounds for the inverted extinction profile by combining Eq. (7), the uncertainty span of $(\gamma, a)$ based on expression (9), and Eqs. (23). Eventually $\alpha_{\text {up }}(R)$ and $\alpha_{\text {low }}(R)$ in inequality (16) were computed as the worst bounds of Eq. (19), i.e., as the ratio of the maximum numerator to the minimum denominator of Eq. (19) for the upper bound and vice versa for the lower bound. Mathematically,

$$
\begin{aligned}
\frac{y_{\text {low }}(R)}{a_{\text {max }}+2 \gamma_{\text {max }} \int_{R}^{R_{m}} y_{\text {up }}(r) \mathrm{d} r} & \leq \hat{\alpha}(R, \gamma, a) \\
\leq & \frac{y_{\text {up }}(R)}{a_{\text {min }}+2 \gamma_{\text {min }} \int_{R}^{R_{m}} y_{\text {low }}(r) \mathrm{d} r},
\end{aligned}
$$

where $a_{\max }=1 / \alpha_{\min }$ and $a_{\min }=1 / \alpha_{\max }$ [Eqs. (18)]. Figure 4 shows an application of this result to the example of Fig. 2. The behavior of absolute bounds warrants some comments.

First, it must be stressed that the bounds are range-dependent error-sensitivity functions of the inverted extinction and that the only inputs needed to compute them are the lidar signal itself and the usererror span [expression (9)], as indicated in Fig. 3. A second point is that performance is strongly dependent on optical depth. Thus, for large optical depths [Fig. 4(a)], divergence of the absolute upper bound occurs and inequality (24) tends to the trivial case of $0<\hat{\alpha}(R, \gamma, a)<\infty$. For moderate-to-low optical depths [Fig. 4(b)], the bounds are always convergent to the upper and lower bounds depicted in Fig. 2 

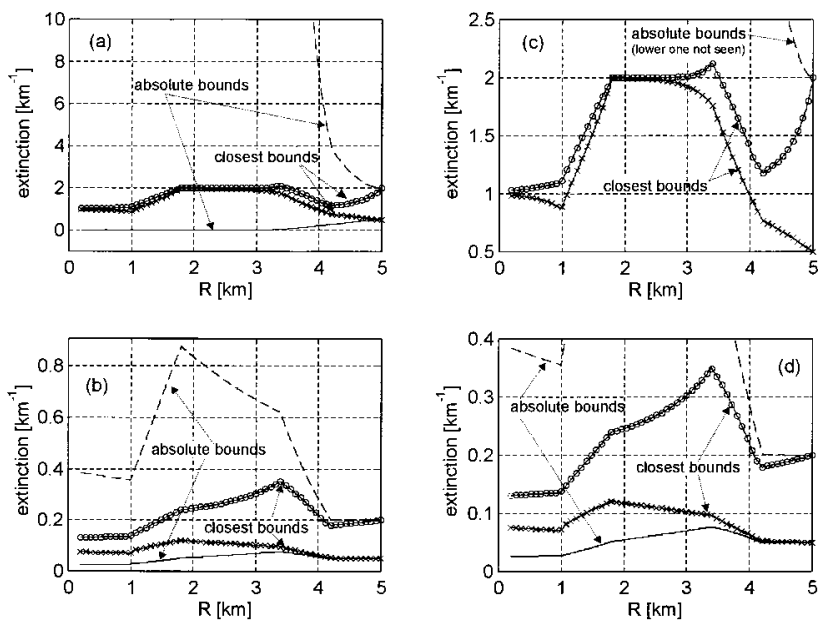

Fig. 4. Trapezium profile. Comparison between the absolute bounds listed in Section 3 and the closest bounds in Section 4 relative to Fig. 2. Subplots (a) and (b), details for $\tau=7.4$ and $\tau=$ 0.74 , respectively. Subplots (c) and (d), reproduction of the closest bounds depicted in Fig. 2 (same scale) with superimposed absolute bounds of subplots (a) and (b) for comparison. Performance of the absolute bounds improves when the optical depths decrease.

(these are the closest bounds and, for the time being, let us assume that they have been obtained by using some graphic procedure). This distinguishing behavior is, in turn, due to that of $x(R)^{\gamma}$. For large optical depths, the range-received power $P(R)$ causes $x(R)$ to span several orders of magnitude along the inversion path. Since the power-law bounds of inequalities (20) are particularly sensitive to $\gamma$ for $x(R)>$ 1 when $x(R)$ is large, a reduced uncertainty in $\gamma$ causes $x(R)^{\gamma_{\min }}$ and $x(R)^{\gamma_{\max }}$ to be fairly different in magnitude. This trend reverses, however, for low optical depths, where the order of magnitude of the normalized return power $x(R)$ is around approximately 1 . Inasmuch as moderate-to-low and low optical depth situations are precisely the ones in which all the extinction branches $\hat{\alpha}\left(R, k_{c}, \alpha_{c}\right)$ move further apart, the absolute bounds assessed in these situations are particularly welcome.

\section{Closest Bounds: Uncertainty Envelope}

The absolute bounds derived in Section 3 are computationally simple but not unique. In fact, they are only some limiting functions, but not the closest ones, of the infinite branches of $\hat{\alpha}(R, \gamma, a)$ when $\gamma$ and $a$ [see Eq. (18)] sweep error intervals of expression (9). Here we tackle the mathematical derivation of such unique bounds, equivalently, the upper and lower envelopes of $\hat{\alpha}(R, \gamma, a)$.

We reconsider Fig. 2 and the already discussed fact that it is not possible to find a single branch belonging to some particular family that behaves as the upper or lower bound of $\hat{\alpha}(R, \gamma, a)$ [Eq. (16)] throughout range $R$. This means that, for each range $R=R_{0}$,

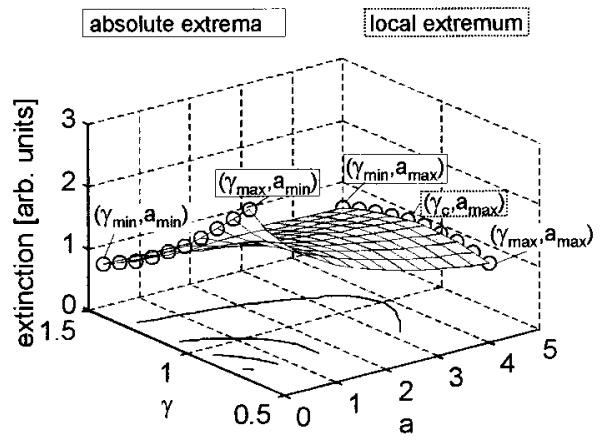

Fig. 5. Absolute and relative extrema of $\hat{\alpha}\left(R_{0}, \gamma, a\right)$ in the feasible domain $(\gamma, a)$. Curves within circles correspond to $\alpha_{\mathrm{fit}}\left(R_{0}, \gamma, a_{\mathrm{min}}\right)$ and $\alpha_{\text {fit }}\left(R_{0}, \gamma, a_{\max }\right)$ [Eq. (31)], for which function $\hat{\alpha}\left(R_{0}, \gamma, a\right)$ always attains its absolute maximum and minimum. In this example, they correspond to corner points $\left(\gamma_{\max }, a_{\min }\right)$ and $\left(\gamma_{\min }, a_{\max }\right)$, respectively within solid boxes). Corner points $\left(\gamma_{\min }, a_{\min }\right)$ and $\left(\gamma_{\max }, a_{\max }\right)$ and local maximum $\left(\gamma_{c}, a_{\max }\right)$ (dotted box $)$ are failed candidates of the absolute extrema. (The plot has been computed from the real live scene shown in Fig. 10, where $R_{0}=497.5 \mathrm{~m}$ ).

optimum pairs $(\gamma, a)$ can be found (i.e., worst-case user-selected pairs),

$$
\left(\gamma_{\text {opt }}{ }^{(\max }, \alpha_{\text {opt }}{ }^{(\max }\right) \quad\left(\gamma_{\text {opt }}{ }^{(\min }, \alpha_{\text {opt }}{ }^{(\min }\right),
$$

for which the inverted extinction

$$
\hat{\alpha}\left(R_{0}, \gamma_{\text {opt }}^{(\max }, \alpha_{\text {opt }}^{(\max }\right)
$$

reaches a maximum value equal to the value of the upper envelope at this range,

$$
\hat{\alpha}\left(R_{0}, \gamma_{\text {opt }}{ }^{(\max }, \alpha_{\text {opt }}{ }^{(\max }\right) \alpha_{\text {up }}\left(R_{0}\right),
$$

and a minimum one, which corresponds to the lower envelope, so that

$$
\hat{\alpha}\left(R_{0}, \gamma_{\text {opt }}{ }^{(\min }, \alpha_{\text {opt }}{ }^{(\min }\right)=\alpha_{\text {low }}\left(R_{0}\right) .
$$

The notation, for example, $\gamma_{\text {opt }}{ }^{\text {(min }}$, should be read as optimum or a worst-case user-selected value of $\gamma$, for which the inverted extinction $\hat{\alpha}$ is minimum (the single left-hand parenthesis is used to distinguish the superscript from a mathematical exponent). Note that by this means we have transformed the problem of finding the upper and lower envelopes of $\hat{\alpha}(R, \gamma, a)$ into an optimization problem with variables $(\gamma, a)$ and that this is equivalent to finding the absolute extrema of this function at each range of $R=R_{0}$.

By use of the classic optimization theory it is known that the extrema (maxima or minima) of a function $f(\gamma, a)=\hat{\alpha}\left(R_{0}, \gamma, a\right)$ in a convex domain (Fig. 5 ), as is the case in expression (9), lie either inside or within the boundary of the domain. Extremum candidates inside the domain are points with $\nabla f=\mathbf{0}$ and those lying within the boundary are formed by both local extrema of the boundary curves and corner points of the domain.

In our case, Eq. (19) has d $\hat{\alpha} / \mathrm{d} a<0$ for all $R$ and $\gamma$ and, therefore, candidate points to absolute extrema must come from a set of candidates formed by local extrema in the boundary curves of the domain $(\gamma$, 
$\left.a_{\max }\right)$ and $\left(\gamma, a_{\min }\right)$, and the four corner points of the domain, $\left(\gamma_{\min }, a_{\min }\right),\left(\gamma_{\min }, a_{\max }\right),\left(\gamma_{\max }, a_{\min }\right)$, and $\left(\gamma_{\max }, \alpha_{\max }\right)$. This obviously involves function evaluation of $\hat{\alpha}(R, \gamma, a)$ at the candidate points as the root (candidate) selection procedure.

Inasmuch as $\mathrm{d} \hat{\alpha} / \mathrm{d} a<0, a_{\mathrm{opt}}{ }^{(\max }=a_{\mathrm{min}}, a_{\mathrm{opt}}{ }^{(\min }=$ $a_{\text {max }}$, and the closest bounds or envelope of $\hat{\alpha}(R, \gamma, a)$ can be written as

$$
\hat{\alpha}\left(R, \gamma_{\text {opt }}^{\min }, a_{\text {max }}\right)<\hat{\alpha}(R, \gamma, a)<\hat{\alpha}\left(R, \gamma_{\text {opt }}^{\max }, a_{\text {min }}\right) .
$$

This relationship represents the optimum branch of families $\hat{\alpha}\left(R, \gamma, a_{\min }\right)$ and $\hat{\alpha}\left(R, \gamma, a_{\max }\right)$, which, at each succeeding range $R=R_{0}$, yields maximum and minimum inverted extinction, respectively, given Eqs. (18) and the user-uncertainty parameters of expression (9), and it is the solution of the posed problem.

Local extrema $\gamma_{c}$, is the domain boundary curves $\left(\gamma, a_{\max }\right)$ and $\left(\gamma, a_{\min }\right)$, can be computed as

$$
\left.\frac{\mathrm{d} \hat{\alpha}}{\mathrm{d} \gamma}\right|_{\substack{a=a_{\max } \\ R=R_{0}}}=0 ;\left.\quad \frac{\mathrm{d} \hat{\alpha}}{\mathrm{d} \gamma}\right|_{\substack{a=a_{\min } \\ R=R_{0}}}=0 .
$$

At this point, note that we distinguish between $\gamma_{c}$ and $\gamma_{\text {opt }}$. The former represents a critical point in the domain of Fig. 5 (i.e., an eligible point to absolute extrema), whereas the latter is an absolute extrema. Equations (26) are the most difficult part of the problem because analytical optimization of Eq. (19) with respect to $\gamma$ involves solution of an integral equation on this parameter for each range $R$. Although, in theory, this formulation of the problem is valid, in practice, the complexity and computational effort involved invalidate the procedure. However, there is a way around the problem if we take into account the reduced error span of $\gamma$ and a Taylor series expansion is used.

First, let us rewrite Eq. (19) as

$$
\hat{\alpha}(R, \gamma, a)=\frac{x(R)^{\gamma}}{a+q(R, \gamma)},
$$

where the integral term is redefined as

$$
q(R, \gamma)=2 \gamma \int_{R}^{R_{m}} x(r)^{\gamma} \mathrm{d} r
$$

Inasmuch as Eq. (26) requires partial derivatives in $\gamma$ for each range of $R=R_{0}$, let us develop $q_{0}(\gamma)=q\left(R_{0}\right.$, $\gamma$ ) in a series expansion around $\gamma=1$ (this is approximately the geometric mean between $\gamma_{\text {min }}$ $=1 / k_{c, \text { max }}=0.75$ and $\left.\gamma_{\max }=1 / k_{c, \text { min }}=1.50\right)$ as

$$
q_{0}(\gamma) \approx 2 \gamma c_{0}+2 \gamma(\gamma-1) c_{1}+\gamma(\gamma-1)^{2} c_{2},
$$

where

$$
c_{n}=\int_{R_{0}}^{R_{m}} x(r) \ln ^{n} x(r) \mathrm{d} r .
$$

Coefficients $c_{n}$ assimilate the morphology of the normalized lidar function around $R=R_{0}, \gamma=1$, and must be recomputed at each point of the inversion interval according to the current data sampling interval (that is to say, we have a different series expansion for each range $R$ ).

Going one step further, if approximation (29) is substituted into Eq. (27) and a second-order series expansion is reused around $\gamma=1$, one can obtain a quadratic fitting function that describes the behavior of $\hat{\alpha}(R, \gamma, a)$ around $R=R_{0}, \gamma=1$. After considerable algebraic manipulation, we found that

$$
\begin{aligned}
\alpha_{\mathrm{fit}}\left(R_{0}, \gamma, a\right)= & \frac{x_{0}}{a+2 c_{0}}+\frac{x_{0}}{a+2 c_{0}}\left(\ln x_{0}-\frac{2\left(c_{0}+c_{1}\right)}{a+2 c_{0}}\right) \\
& \times(\gamma-1)+\frac{x_{0}}{a+2 c_{0}}\left[1 / 2 \ln ^{2} x_{0}\right. \\
& -\frac{\left(2 c_{1}+c_{2}\right)}{a+2 c_{0}}-\left(\ln x_{0}-\frac{2\left(c_{0}+c_{1}\right)}{a+2 c_{0}}\right) \\
& \left.\times \frac{2\left(c_{0}+c_{1}\right)}{a+2 c_{0}}\right](\gamma-1)^{2}+O\left[(\gamma-1)^{3}\right],
\end{aligned}
$$

where $x_{0}=x\left(R_{0}\right)$.

Two of these functions, $\alpha_{\mathrm{fit}}\left(R_{0}, \gamma, a_{\min }\right)$ and $\alpha_{\mathrm{fit}}\left(R_{0}\right.$, $\gamma, a_{\text {max }}$ ), are plotted in Fig. 5 as curves within circles. They fit the sensitivity of the inverted extinction profile at $R=R_{0}$ with $\gamma$ for two boundary extinction calibrations located within the limits of the uncertainty domain, $a=a_{\text {min }}$ and $a=a_{\text {max }}$. Critical points of $\hat{\alpha}(R, \gamma, a)$ always lie on these boundary curves. A combination of Eqs. (26) and (31) yields a pair of first-order equations in $\gamma$ (one for each derivative), after which local extrema $\gamma_{c, 1}$ and $\gamma_{c, 2}$ can easily be solved.

Eventually, since candidate points to absolute extrema $\gamma_{\text {opt }}{ }^{\max } \gamma_{\mathrm{opt}}{ }^{(\min }$ are formed by the corner points plus the local extrema that lie inside the uncertainty intervals of expression (9), the sought-after bounds for the inverted extinction can be computed as

$$
\begin{gathered}
\left\{\begin{array}{c}
\alpha_{\text {up }}(R)=\max \left[\hat{\alpha}\left(R, \gamma_{i}, a_{\min }\right)\right] \\
\alpha_{\text {low }}(R)=\min \left[\hat{\alpha}\left(R, \gamma_{i}, a_{\text {max }}\right)\right]
\end{array}\right. \\
\gamma_{i}=\gamma_{\max }, \gamma_{\min }, \gamma_{c, 1}(R), \gamma_{c, 2}(R) .
\end{gathered}
$$

The functional dependence of $\gamma_{c}$ with range $R$ is a reminder that local extrema must be recomputed at each range of $R=R_{0}$.

Equation (32) is the solution of expression (25). Even though the computational load per inversion point of the procedure is virtually nil with the computational tools available today, Eq. (32) adds up to six function evaluations [four in Eq. (32) and two more to solve the first-order equation pair derived with Eqs. (26) and (31)] plus computation of three numerical integrals [coefficients $c_{0}, c_{1}$, and $c_{2}$ in Eq. (30)]. Contrary to what happens with the corner points, the main drawback of the calculation of $\gamma_{c, 1}$ 
and $\gamma_{c, 2}$ stems from the fact that they must be recomputed at each succeeding range $R$. Although still computationally efficient because Eq. (30) can easily be vectorized, this makes the algorithm less straightforward.

Considerable work in the form of reconsideration of Eq. (31) for both simulated and real data sets has proved two points.

(1) When computation of local candidates $\gamma_{c, 1}\left(R_{0}\right)$ and $\gamma_{c, 2}\left(R_{0}\right)$ is neglected, negligible error results (see Section 5) for all the situations studied. Note that this results in substantial simplification of the procedure outlined above because it leaves only the four corner points as the only valid candidates and these points do not need to be recomputed at each range $R$ because they are constants that define the span of the feasible domain. Examples are discussed in Section 5 .

(2) Expansion of Eq. (31) to higher orders is illadvised, because it is necessary to strike a balance between the improvement in accuracy of $a_{\mathrm{fit}}(R, \gamma, a)$ and the complexity increase in the root selection procedure. Even though this enabled us to fit more complicated shapes of $\hat{\alpha}(R, \gamma, a)$ with $\gamma$ and, therefore, to output more local candidates, in practice, most of them lie outside the feasible domain [expression (9)] or correspond to inflection points. For most of the situations studied, $\hat{\alpha}(R, \gamma, a)$ resembles an inclined plane and even shapes similar to those in Fig. 5 are less frequent. In other words, considering the reduced span of $\gamma$, the shape of $\hat{\alpha}(R, \gamma, a)$ is not more complicated than the one shown in Fig. 5.

\section{Quantification of the Closeness Error}

To quantify numerically the performance of the bounds inverted in Sections 3 and 4, we define the closeness error, $\epsilon(R)$, for the upper bound as

$$
\epsilon_{\mathrm{up}}(R)=\frac{\alpha_{\mathrm{up}}(R)-\max \{\alpha(R, \gamma, \alpha)\}}{\max \{\alpha(R, \gamma, \alpha)\}} .
$$

For each range $R, \epsilon_{\text {up }}(R)$ is computed as the relative difference between the upper bound, $\alpha_{\text {up }}(R)$, and the upper envelope of all the inverted extinction families. If, with the aid of a computer, a sufficiently large number of families are available, this expression becomes representative of the closeness error. Likewise,

$$
\epsilon_{\mathrm{low}}(R)=\frac{\alpha_{\mathrm{low}}(R)-\min \{\alpha(R, \gamma, a)\}}{\min \{\alpha(R, \gamma, a)\}}
$$

estimates the closeness error for the lower bound, $\alpha_{\text {low }}(R)$. Application of this expression might, however, become difficult for low extinctions because Eq. (34) becomes the ratio of two small numbers and, as a result, peaking might occur in the plot of $\epsilon_{\text {low }}$ versus range.

Alternatively, the ratio of the error interval between the upper and the lower bounds to the thickness of the envelope of all the family curves can be used as a likelihood function to quantify composite performance of the bounds. This formulation copes with the sensitivity problem of Eq. (34) for low extinctions. It can be expressed as

$$
\Lambda(R)=\frac{\left|\alpha_{\mathrm{up}}(R)-\alpha_{\mathrm{low}}(R)\right|}{\max \{\alpha(R, \gamma, a)\}-\min \{\alpha(R, \gamma, a)\}} .
$$

This ratio tends to unity as long as the bounds tend to coincide with the upper and lower envelopes of all the families. Then, the error function $\Lambda(R)-1$ tends to zero.

An often more convenient way of assessing closeness errors is, however, to compute Eqs. (33)-(35) in a scalar fashion rather than as range-dependent functions. One can achieve this by using the customary error-norm definition.

$$
\|\epsilon\|=\left[\frac{1}{N} \sum_{i=1}^{N} \epsilon\left(R_{i}\right)^{2}\right]^{1 / 2} .
$$

In the case of the likelihood function $\Lambda(R)-1$ is computed as the scalar $\|\Lambda\|-1$.

Without the need to use these error expressions, simple visual cross examination of Figs. 2 and 4 is enough to reveal outstanding performance of the closest bounds of Section 4, even when local extrema $\left[\gamma_{c, 1}\left(R_{0}\right)\right.$ and $\gamma_{c, 2}\left(R_{0}\right)$ from Eqs. (26) and (31)] are not taken into account. Analytical justification of this approximation, for example, in terms of the optical depth, is difficult because coefficients $c_{n}(R)$ in Eq. (30) depend on the morphology of the optical return and, eventually, on physical parameters of the aerosols.

Until now, the inversion examples of Fig. 2 and related bounds of Fig. 4 have relied on the inversion of power returns synthesized from a common shape of the extinction profile (the trapezium platform described in Section 2), which is a slow-varying function with range. From the point of view of the morphology of the function and with a view to assessing closeness errors as comprehensively as possible, it is interesting to extend our simulations to fast-varying input extinction profiles as well and to parameterize the simulations for different inversion ranges (not necessarily from 0.2 to $5 \mathrm{~km}$ as we have done so far) and optical depths. These criteria build the core of 18 parametric simulations that will be discussed next. Afterward, we will be able to assess closeness errors.

Fast-varying scalable extinction profiles have been devised by use of an affine transformation of the form

$$
\alpha(R)=\bar{\alpha} f(a R+b),
$$

where $f$ is a unity-mean fast-varying function, $a$ and $b$ are scaling parameters, and $\bar{\alpha}$ is the mean extinction of $\alpha(R)$. $f$ was constructed as a two-hump profile with superimposed frequency varying ringing of the form

$$
f(t)=g(t)+c \sin [\mathrm{d} \operatorname{tg}(t)]+e,
$$



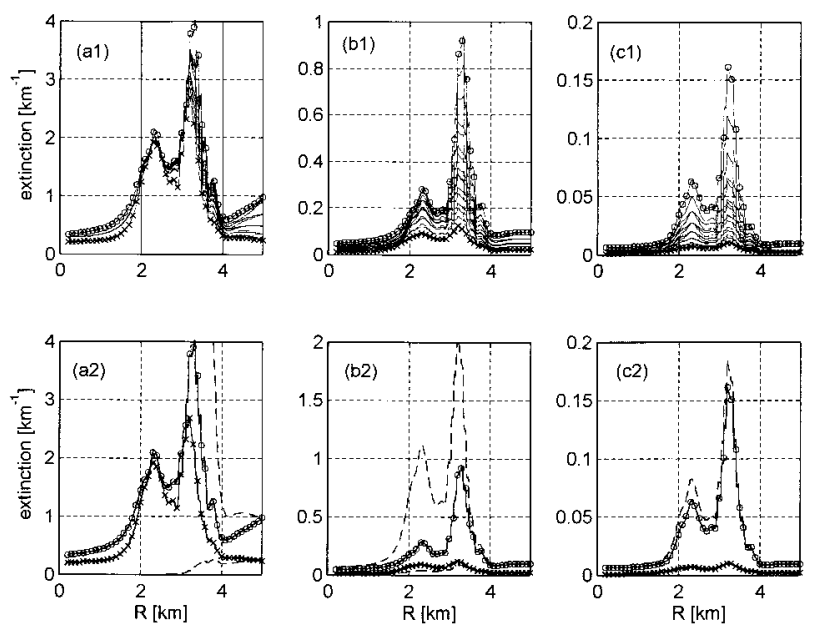

Fig. 6. Parametric study versus optical depth similar to that in Figs. 2 and 4, with Eq. (37) as the common shape of the extinction profile: (a) $\tau=4.9$, (b) $\tau=0.49$, (c) $\tau=0.049$ (a1)-(c1) show families of inverted solutions with superimposed closest bounds: $\bigcirc$, upper bounds; $\times$, lower bounds. (a2)-(c2) show related error bounds: closest bounds as indicated; absolute bounds represented by a dashed curve). This is case study (3) in Table 1.

$$
g(t)=g_{0}\left[\frac{1}{\left(t-t_{0}\right)^{2}+t_{1}}+\frac{1}{\left(t-t_{2}\right)^{2}+t_{3}}\right]
$$

where $c, d, e, g_{0}, t_{0}, t_{1}, t_{2}$, and $t_{3}$ are characteristic shape parameters.

Figure 6, which is the fast-varying counterpart of Fig. 2, illustrates a parametric study for optical depths in the $\tau=0.049-4.9$ range by use of the Eq. (37) profile. Figures 6(a1)-6(c1) depict inverted extinction families given the user-error margin $\alpha_{m} / 2 \leq$ $\alpha_{c} \leq 2 \alpha_{m}$ and $0.67 \leq k_{c} \leq 1.34$ [expression (9)] for decreasing optical depths and Figs. 6(a2)-6(c2) plot associate error bounds as in Fig. 4. The closeness error functions [Eqs. (33) and (34)] for the absolute bounds plotted in Figs. 6(a2)-6(c2) (dashed curves) are shown in Fig. 7. As a result, we reencounter the same behavior for fast-varying functions as we found in Section 3 for slow-varying functions: closeness errors $\epsilon_{\mathrm{up}}(R)$ and $\epsilon_{\mathrm{low}}(R)$ progressively reduce as long as the optical depth decreases. In particular, convergence is achieved only in cases (b) and (c) $(\tau=0.49$ and 0.049 , respectively).

Figure 8 summarizes the closeness error of the 18 parametric simulations introduced above, which include all the examples presented so far. The two examples comprising slow- and fast-varying profiles (Figs. 2, 4, and 6, respectively) have been repeated now for the three cases listed in Table 1 and, in turn, each case study has been repeated for three optical thicknesses separated by a factor of 10 . As a result, we get two simulation sets: slow-varying or trapezium profile and fast-varying or the Eq. (37) profile, each set comprising nine simulations. In contrast with Fig. 7, Fig. 8 plots the scalar closeness error or per-unit norm-error [Eq. (36)]. Of course, closeness
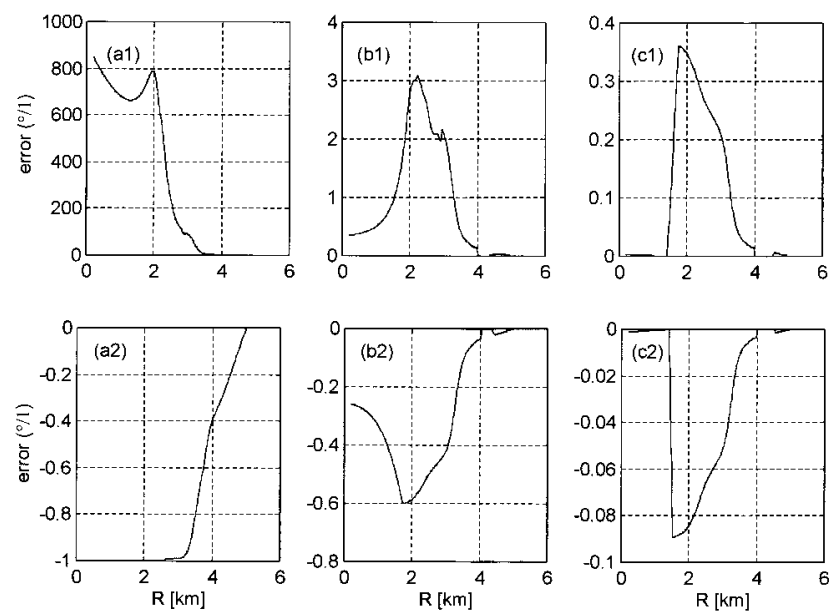

Fig. 7. Parametric study of the closeness error for the absolute bounds illustrated in Figs. 6(a2)-6(c2). Here, (a1)-(c1) show $\epsilon_{\text {up }}(R)$ [Eq. (33)] and (a2)-(c2) show $\epsilon_{\text {low }}(R)$ [Eq. (34)]. Note that $\epsilon(R)$ is computed as a per-unit variation.

errors for the error bounds retrieved in our previous examples and shown in Figs. 4 and 6(a2)-6(c2) can be identified by label (3) in Table 1 and the corresponding optical thickness $\tau$ in Fig. 8.

Judging by Fig. 8, performance of the closest bounds is usually much better than that of the absolute bounds, as expected, with $\left\|\boldsymbol{\epsilon}_{\text {up }}\right\|$ seldom exceeding some $0.1 \%$ and $\left\|\epsilon_{\text {low }}\right\|$ being virtually nil. $\|\Lambda\|-1$ (not shown) is always below $-10^{-3}(-0.1 \%)$ and usually
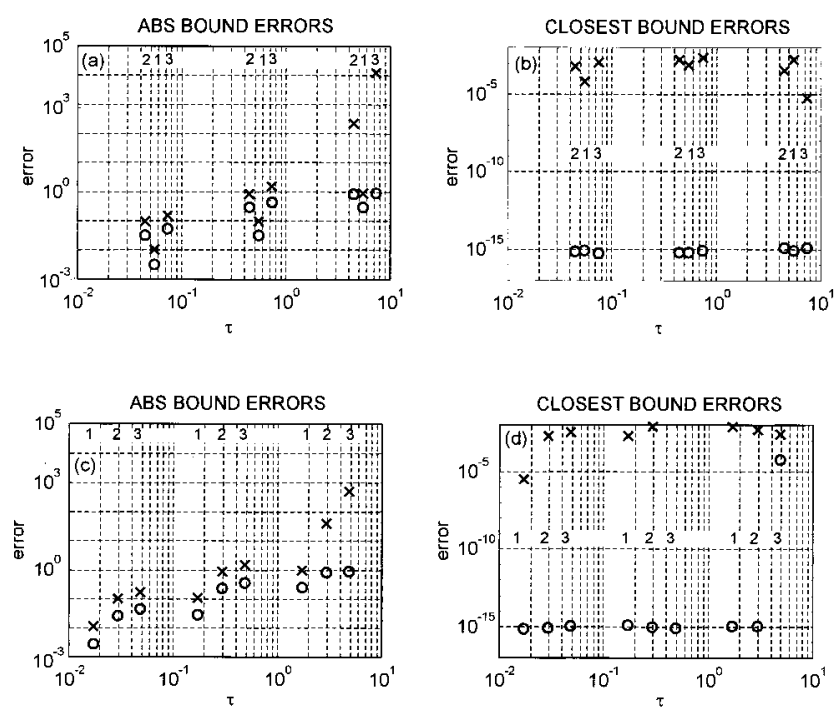

Fig. 8. Comparative plots showing closeness errors of the absolute and closest bounds (Sections 3 and 4, respectively) for optical depths in the range of $\tau=0.01-10$ and by use of two different extinction scenes as inputs: (a), (b) trapezium profile; (c), (d) Eq. (37) profile: $\times$ closeness error $\left\|\epsilon_{\text {up }}\right\|$ [Eqs. (33) and (36)]; $\bigcirc,\left\|\epsilon_{\text {low }}\right\|$ [Eq. (34) and (36)]; labels 1, 2, and 3 are explained in Table 1. In all cases, performance of the closest bounds is much better than that of the absolute bounds with $\left\|\epsilon_{\text {up }}\right\|$ typically well below $10^{-3}$ $(0.1 \%)$ and $\left\|\epsilon_{\text {low }}\right\|$ virtually negligible. For the absolute bounds, the results are also much more dependent on optical thickness. 
Table 1. Simulation Ranges and Labels for Figs. 8 and 9

\begin{tabular}{cccc}
\hline Label & $\begin{array}{c}\text { Minimum Range } \\
\mathrm{R}_{\min }(\mathrm{m})\end{array}$ & $\begin{array}{c}\text { Maximum Range } \\
\mathrm{R}_{\max }(\mathrm{m})\end{array}$ & $\begin{array}{c}\text { Resolution } \\
\Delta \mathrm{R}(\mathrm{m})\end{array}$ \\
\hline$(1)$ & 5.000 & 5.300 & 1 \\
$(2)$ & 10 & 300 & 1 \\
$(3)$ & 200 & 5.000 & 10 \\
\hline
\end{tabular}

falls to some $10^{-15}$ round-off numerical error floor. In principle, application of the closest bounds is thus possible for any optical depth, without any other main limitations. In practice, application of the closest bounds is limited only by the extent of validity of the aerosol-dominant backward inversion method of Eq. (7), the power-law relationship of Eq. (4), and the single-scatter lidar equation (1). Convergence of the absolute bounds is, however, restricted to optical depths $\tau<1$ and practical results are usually obtained for $\tau<0.1$ (this yields closeness errors of some $30 \%$ for the upper bound and less than $10 \%$ for the lower bound).

\section{Errors on the Retrieved Optical Thickness}

Similar to Fig. 8, Fig. 9 plots over estimation and underestimation errors $\left(\epsilon_{\tau, \max }\right.$ and $\epsilon_{\tau, \min }$, respectively) on the retrieved optical thickness for the two simulation sets considered. These errors are defined as

$$
\begin{gathered}
\epsilon_{\tau, \max }=\frac{\tau\left(\alpha_{\mathrm{up}}\right)-\tau_{0}}{\tau_{0}}, \\
\epsilon_{\tau, \text { min }}=\frac{\tau_{0}-\tau\left(\alpha_{\mathrm{low}}\right)}{\tau_{0}},
\end{gathered}
$$

where $\tau\left(\alpha_{\text {up }}\right)$ and $\tau\left(\alpha_{\text {low }}\right)$ are the computed optical thicknesses obtained with Eq. (13) by use of the upper closest bound $\alpha_{\text {up }}(R)$ and the lower bound, $\alpha_{\text {low }}(R)$ as extinction functions [Eq. (32)], respectively, and $\tau_{0}$ is the nominal optical thickness of the synthesized profile (i.e., without calibration errors or, equivalently, with ideal parameters $k$ and $\alpha_{m}$ ). Note that both Eqs. (39) and (40) are defined as positive quantities and can be interpreted as overestimation and underestimation errors, respectively.

Inspection of the plots in Fig. 9 reveals two important points:
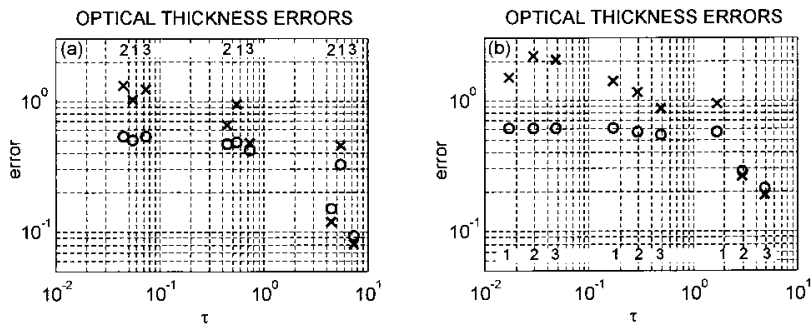

Fig. 9. Error margin on the retrieved optical thickness for the two simulation sets studied: (a) trapezium profile input, (b) Eq. (37) profile input; $\times \epsilon_{\tau, \max }$ [Eq. (39)]; $\bigcirc, \epsilon_{\tau, \min }$ [Eq. (40)]; labels 1, 2 and 3 are explained in Table 1.

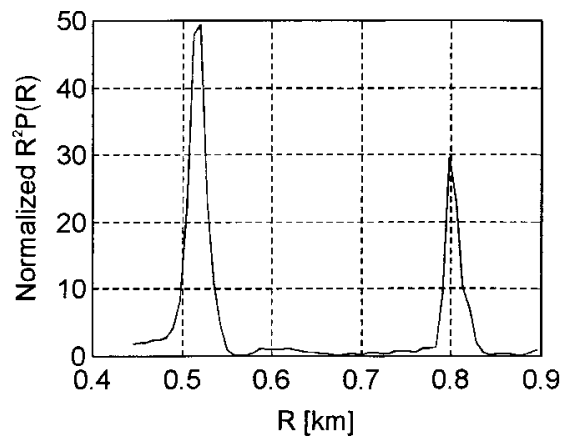

Fig. 10. Normalized $R^{2}$-corrected function $x(R)$ corresponding to a real inversion example (445-895 m). The profile shows two storm cloud layers located between 450 and $550 \mathrm{~m}$ and between 785 and $835 \mathrm{~m}$.

One is that errors in the retrieved optical thickness reduce for increased optical thickness. This is in agreement with point (1) of Section 2.

The other comes from cross examination of subplots (a) and (b) in Fig. 9. Errors $\epsilon_{\tau, \max }, \epsilon_{\tau, \min }$, and the error margin $\left|\epsilon_{\tau, \max }-\epsilon_{\tau, \min }\right|$ as well (i.e., the distance between $\times$ and $\bigcirc$ for a given $\tau$ ) are lower for slow-varying functions [Fig. 9(a)] than for fastvarying functions [Fig. 9(b)]. As a result, if we compare performance of $\epsilon_{\tau, \max }$ in both plots, the different morphology of the extinction function represents approximately a factor of 2 difference (this can best be seen for $\tau<1$ ). Although this is inferred from only two sets (nine simulations each), this figure might be extrapolated to a sensitivity figure of the closest bounds in front of different input atmospheric scenes.

\section{Application to a Live Scene}

As a final example to demonstrate to the reader that the theory developed can be used freely to play with the real-world range of slow- and fast-varying extinction profiles and optical depths, Figs. 10 and 11 illustrate its application to a real lidar return from storm clouds. In this case, the procedure in Section 4 has been computed twice in all the cases, once including computation of local extrema [Eqs. (26)-(31)] and once taking into consideration corner points only without significant differences. As already discussed in Subsection 1.A, it is widely acknowledged that the assumption of a constant extinction-tobackscatter ratio and exponent term $k$ over all the inversion range is an oversimplification of the problem and that accurate inversion would certainly require convenient partitioning of the inversion range into appropriate subintervals, ${ }^{31}$ each with a particular pair of $B$ and $k$ [Eq. (4)].

We collected live data using a Nd:YAG elastic backscatter lidar system. The 10-ns-long laser emitted $390 \mathrm{~mJ}$ of energy, produced $10-\mathrm{Hz}$ repetition rate pulses, was equipped with a 12 -bit $20-\mathrm{MHz}$ digitizer, and was used to obtain sample points spaced $7.5 \mathrm{~m}$ apart over the lidar return. The field of view of the receiving optics was $0.2 \mathrm{mrad}$ so that only singlescattered components of the backscattered laser ra- 

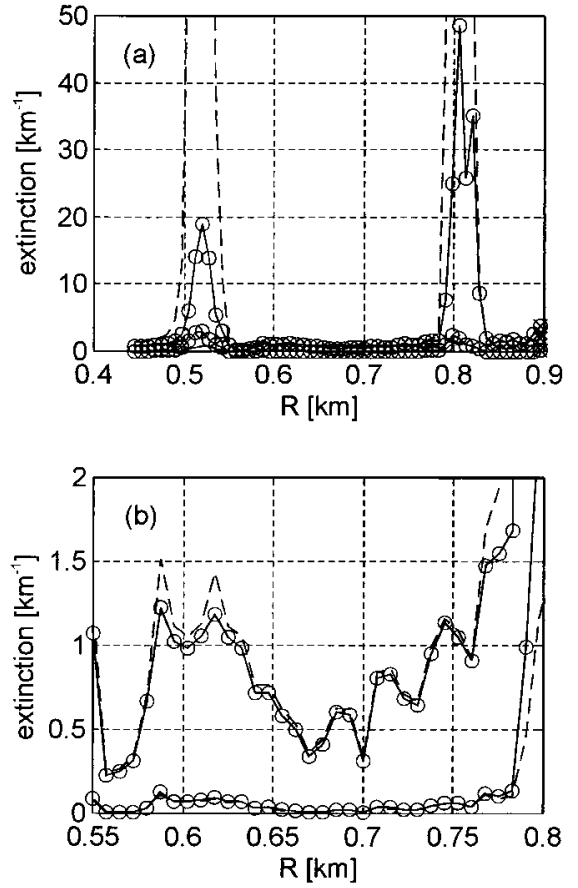

Fig. 11. Error bounds for the live scene of Fig. 10: (a) comparison between the absolute bounds of Section 3, Eq. (24) (dashed curves) and the closest bounds of Section 4, Eq. (32) (curves with circles). (b) Zoom-in detail of the range interval between the two cloud layers (550 and $800 \mathrm{~m}$ ).

diation were detected. The normalized $R^{2}$-corrected return, $x(R)$, is shown in Fig. 10. It was formed by averaging 40 return pulses in the range interval between 445 and $895 \mathrm{~m}$. This averaging factor was adjusted by trial and error and was shortened to the temporal correlation length of the atmosphere (i.e., stationarity can be assumed). The interval shows two dense water cloud layers, the first between 450 and $550 \mathrm{~m}$ and the second between 785 and $835 \mathrm{~m}$. Since the cloud is optically thick, an estimate of the boundary extinction $\left[\alpha_{m}\right.$ in Eq. (6)] at $895 \mathrm{~m}$ was computed from application of the exponential curvefitting method of Eq. (8) to the end interval between 850 and $895 \mathrm{~m}$, which yielded $\alpha_{c} \approx 2.0 \mathrm{~km}^{-1}$.

For computation of the error bounds, we followed the methodology used in the preceding examples, five inversion families (three branches each) were inverted from $x(R)$ using the same error percentages ( $\pm 90 \%$ for $\alpha_{c}$ or, equivalently, $\alpha_{c}=0.2,1.1,2.0,2.9$, and $3.8 \mathrm{~km}^{-1}$ and $0.67-1.34$ for $k$ ) for comparative purposes. Absolute and closest extinction bounds are plotted in Fig. 11(a), and Fig. 11(b) expands the range interval $(550-800 \mathrm{~m})$ between the two cloud layers.

With respect to the computed closeness error for the absolute bounds we obtained $\left\|\boldsymbol{\epsilon}_{\text {up }}\right\|=3.4,\left\|\boldsymbol{\epsilon}_{\text {low }}\right\|=$ 0.37 , and $\|\Lambda\|-1=5.7$. These figures are in good agreement with what we assessed in Fig. 8(c) for fast-varying functions $x(R)$ [equivalently, fastvarying extinction profiles such as in Eq. (37)]. The same figures for the closest bound errors are com- pletely negligible $\left(<10^{-15}\right)$, and again we retrieved higher errors for the upper bound than for the lower bound.

To estimate errors in the retrieved optical thickness, the closest bounds inverted in Fig. 11, $\alpha_{\text {up }}(R)$ and $\alpha_{\text {low }}(R)$, yielded an optical thickness range between $\tau=0.55$ and $2.4(T=-4.8$ and $-20.6 \mathrm{~dB})$. When we computed nominal thickness $\tau_{0}$ from Eq. (13) with $\alpha(R)=\alpha\left(R, k_{c}, \alpha_{c}\right)$ and $k_{c}=1$ and $\alpha_{c}=2$ $\mathrm{km}^{-1}$ (i.e., we computed the inverted extinction from the user guesses), $\tau_{0}=1.4(-11.9 \mathrm{~dB})$ was obtained. Eventually, this translates into $\epsilon_{\tau, \max }=0.74$ and $\epsilon_{\tau, \min }=0.60$, which is also in accordance with the error assessment plot of Fig. 11(b).

\section{Conclusions}

We departed from the lidar backward inversion algorithm that yields a stable inhomogeneous solution of the lidar equation and focused on the development of an error sensitivity study that enables one to relate errors in the user-input parameters boundary extinction and exponential term in the extinction-tobackscatter relationship, $\left(k_{c}, \alpha_{c}\right)$, to errors in the inverted extinction profile. The choice of these particular parameters has been based on the customary approach, whereby backscatter and extinction profiles are related by a power-law relationship [Eq. (4)], which has been proved to be of practical value. ${ }^{20}$ We tested the validity of the study for optical depths by extensive simulation in the range $\tau=0.01-10$. Hence, the theory we developed can be used freely to play with the real-world range of extinction and optical depths without any limitations on the extent of the valid backward inversion algorithm itself [particularly, Eqs. (4) and (7)] at these optical depths.

As introductory background, we have shown that, when parameters $\left(k_{c}, \alpha_{c}\right)$ (equivalently, $\gamma=1 / k_{c}$ and $a=1 / \alpha_{c}$ ) take any possible value from the predetermined user-defined uncertainty interval, a set of inverted extinction families $\hat{\alpha}(R, \gamma, a)$ can be obtained, all of which are solutions of the lidar equation. Although this is not new, it has allowed us to derive analytical sensitivity studies that relate the inverted set of extinction profiles to atmospheric profiles. Yet, the core of this work has been devoted to the derivation of range-dependent error functions or bounds of $\hat{\alpha}(R, \gamma, a)$, (in Sections 3 and 4), which has enabled us to compute the range-dependent error span of the inverted extinction with (1) the user-error span in the sensitivity parameters and (2) the elastic backscatter optical return. This has been done in two main steps with progressively improved accuracy:

First, in the case of absolute bounds, some upper and lower envelopes of the inverted families $\hat{\alpha}(R, \gamma, a)$ have been found but are not necessarily the closest bounds. It has been shown that the closeness error reduces for moderate-to-low optical depths and that the performance of the lower absolute bound is always much better than that of the upper bound.

The second step comprises derivation of a mathematical procedure that yields the sought-after closest 
possible bounds (i.e., with a closeness error that is virtually nil). These are the best bounds. The key of the procedure is the appropriate transformation of the initial problem into an equivalent optimization with variables $(\gamma, a)$, whose feasible domain is the user-uncertainty span. Further refinement has led to a simpler and more straightforward method with closeness errors around an encouraging $0.1 \%$. This has reduced the procedure to four function evaluations of $\hat{\alpha}(R, \gamma, a)$ (the corner points of the feasible domain) per inversion cell. Simulations and the inversion of a live return have also shown that excellent performance is maintained for both slow- and fastvarying profiles.

All things considered, since the results obtained here can be perfectly applied to successive intervals of the atmosphere as in the so-called slice method of inversion, we are certain that the closest bounds procedure will be a long-lasting practical contribution of this research to the field of lidar inversion.

\section{Appendix: Relationship between the Inverted and the True Extinction Profiles}

Assume that $k_{c}$ and $\alpha_{c}$ are the user-selected values for the exponential term in the backscatter-to-extinction ratio of Eq. (4) and the boundary extinction of Eq. (6), respectively, and that $k$ and $\alpha_{m}$ are their true atmospheric counterparts. Therefore, the user-inverted extinction profile corresponds to the backward solution of Eq. (7) except for the replacement of $k$ by $k_{c}$ and $\alpha_{m}$ by $\alpha_{c}$ as follows:

$$
\hat{\alpha}\left(R, k_{c}, \alpha_{c}\right)=\frac{\exp \left[\left(S-S_{m}\right) / k_{c}\right]}{\alpha_{c}{ }^{-1}+\frac{2}{k_{c}} \int_{R}^{R_{m}} \exp \left[\left(S-S_{m}\right) / k_{c}\right] \mathrm{d} r} .
$$

By substituting the basic single-scatter lidar Eq. (1) into Eq. (17), the normalized $R^{2}$-corrected return power, $x(R)$, takes the form

$$
x(R)=\exp \left(S-S_{m}\right)=\frac{\alpha(R)^{k} \exp \left[-2 \int_{R_{0}}^{R}(\alpha(r) \mathrm{d} r]\right.}{\alpha_{m}{ }^{k} \exp \left[-2 \int_{R_{0}}^{R_{m}} \alpha(r) \mathrm{d} r\right]},
$$

where $R_{m}$ is the maximum range and $R_{0}$ is some predetermined minimum range, which is irrelevant in Eq. (10).

If Eq. (A2) is substituted into Eq. (A1) and the auxiliary functions defined in Eqs. (11) and (12) are reconsidered, we obtain the sought-after solution of Eq. (10).

When the error in the exponential term of the extinction-to-backscatter ratio can be assumed to be negligible (i.e., the approximation $k_{c} \approx k$ holds true) and only the boundary extinction calibration error is of interest, it is possible to derive a simple error sen- sitivity relationship that links the relative error in such a parameter to the inverted extinction profile.

We depart from the equivalent form of Eq. (12), and we substitute Eq. (11) into Eq. (12). When we use $k_{c}=k$,

$$
F(R, \boldsymbol{\alpha})=\int_{R}^{R_{m}} \alpha(r) \exp \left\{-\frac{2}{k} \int_{R_{0}}^{r} \alpha(z) \mathrm{d} z\right\} \mathrm{d} r .
$$

Equation (A3) can be integrated by use of the substitution

$$
u(r)=\exp \left\{-\frac{2}{k} \int_{R_{0}}^{r} \alpha(z) \mathrm{d} z\right\}
$$

so that it yields

$$
F(R, \boldsymbol{\alpha})=\frac{k}{2}\left[I(R, k)-I\left(R_{m}, k\right)\right] .
$$

Eventually, substitution of $k=k_{c}$ and Eq. (A5) into Eq. (10) would yield the simple form

$$
\hat{\alpha}\left(R, k, \alpha_{c}\right)=\frac{\alpha(R)}{1+\left(\frac{1}{\epsilon_{c}}-1\right) \frac{I\left(R_{m}, k, \boldsymbol{\alpha}\right)}{I(R, k, \boldsymbol{\alpha})}} ; \quad \epsilon_{c}=\frac{\alpha_{c}}{\alpha_{m}} .
$$

Equation (A6) relates the user-inverted extinction profile $\hat{\alpha}\left(R, k, \alpha_{c}\right)$ to the true atmospheric extinction profile $\alpha(R)$ (equivalently, $\boldsymbol{\alpha}$ in vector form) as a function of the relative error in the calibration of the boundary extinction $\epsilon_{c}$. It is advantageous to use Eq. (A6) for simulation purposes when a computergenerated atmospheric profile $\alpha(R)$ is available and an error sensitivity study is performed as discussed in the preceding sections.

In the conclusion of Section 2 we stipulated that overestimation of the boundary extinction $\alpha_{m}$ is usually preferred to underestimation because it can be easily corroborated by taking into account the amplification error term $\left(1 / \epsilon_{c}-1\right)$ in Eq. (A6). Thus, for example, $\alpha_{c}=10 \alpha_{m}$ yields an error amplification of only -0.9 , whereas for $\alpha_{c}=0.1 \alpha_{m}$ the error amplification becomes ten times larger.

We acknowledge the sponsorship of the Interministry Committee for Science and Technology under grants AMB96-C02-C01 and TIC431-93.

\section{References}

1. W. Hitschfeld and J. Bordan, "Errors inherent in the radar measurement of rainfall at attenuating wavelengths," J. Appl. Meteorol. 11, 58-67 (1954).

2. E. W. Barret and O. Ben-Dov, "Application of the lidar to air pollution measurements," J. Appl. Meteorol. 6, 500-515 (1967).

3. W. Viezee, E. E. Uthe, and R. T. H. Collis, "Lidar observations of airfield approach conditions: an exploration study,“ J. Appl. Meteorol. 8, 274-283 (1969).

4. P. A. Davis, "Analysis of lidar signatures of cirrus clouds," Appl. Opt. 8, 2099-2102 (1969).

5. F. G. Fernald, B. M. Herman, and J. A. Reagan, "Determina- 
tion of aerosol height distribution by lidar," J. Appl. Meteorol. 11, 482-489 (1972).

6. R. T. H. Collis and P. B. Russell, "Lidar measurement of particles and gases by elastic backscattering and differential absorption," in Laser Monitoring of the Atmosphere, E. D. Hinkley, ed. (Springer-Verlag, New York, 1976), Chap. 4, pp. 71-102.

7. R. H. Kohl, "Discussion of the interpretation problem encountered in single-wavelength lidar transmissometers," J. Appl. Meteorol. 17, 1034-1038 (1978).

8. J. D. Klett, "Stable analytical inversion solution for processing lidar returns," Appl. Opt. 20, 211-220 (1985).

9. S. R. Pal, W. Steinbrecht, and A. I. Carswell, "Automated method for lidar determination of cloud-base height and vertical extent," Appl. Opt. 31, 1488-1494 (1992).

10. G. J. Kunz and G. de Leeuw, "Inversion of lidar signals with the slope method," Appl. Opt. 32, 3249-3256 (1993).

11. F. Rocadenbosch, A. Comerón, and D. Pineda, "Assessment of lidar inversion errors for homogeneous atmospheres," Appl. Opt. 37, 2199-2206 (1998).

12. G. J. Kunz, "Vertical atmospheric profiles measured with lidar," Appl. Opt. 22, 1955-1957 (1983).

13. J. A. Ferguson and D. H. Stephans, "Algorithm for inverting lidar returns," Appl. Opt. 22, 3673-3675 (1983).

14. F. G. Fernald, "Analysis of atmospheric lidar observations: some comments," Appl. Opt. 23, 652-653 (1984).

15. Y. Sasano, "Observational study on atmospheric mixed layer and transition layer structures using Mie lidar," J. Meteorol. Soc. Jpn. 63, 419-435 (1985).

16. H. G. Hughes, J. A. Ferguson, and D. H. Stephans, "Sensitivity of a lidar inversion algorithm to parameters relating atmospheric backscatter and extinction,” Appl. Opt. 24, 1609-1613 (1985).

17. J. D. Klett, "Extinction boundary value algorithm for lidar inversion," Appl. Opt. 25, 2462-2464 (1986).

18. L. R. Bissonnette, "Sensitivity analysis of lidar inversion algorithms," Appl. Opt. 25, 2122-2125 (1986).

19. Y. Sasano and H. Nakane, "Significance of the extinction backscatter ratio and the boundary value term in the solution for the two-component lidar equation," Appl. Opt. 23, 11-13 (1984).

20. J. D. Klett, "Lidar inversion with variable backscatter extinction ratios," Appl. Opt. 24, 1638-1643 (1985).

21. M. Kaestner, "Lidar inversion with variable backscatter/extinction ratios: comment," Appl. Opt. 25, 833-835 (1986).

22. Y. Sasano, E. V. Browell, and S. Ismail, "Error caused by using a constant extinction/backscattering ratio in the lidar solution," Appl. Opt. 24, 3929-3932 (1985).

23. R. G. Pinnick, J. M. Rosen, D. J. Hofman, "Stratospheric aerosol measurements. III: Optical model calculations, “ J. Atmos. Sci. 33, 304-314 (1976).

24. P. B. Russell, T. J. Swissler, M. P. McCormick, W. P. Chu, J. M. Livingston, and T. J. Pepin, "Satellite and correlative measurements of the stratospheric aerosol. I: An optical model for data conversion," J. Atmos. Sci. 38, 1279-1294 (1981).

25. V. E. Zuev, "Laser beams in the atmosphere," translated from the Russian by J. S. Wood (Consultants Bureau, New York, 1982).

26. Y. Sasano and H. Nakane, "Quantitative analysis of RHI lidar data by an iterative adjustment of the boundary condition term in the lidar solution," Appl. Opt. 26, 615-616 (1987).

27. R. W. Fenn, "Correlation between atmospheric backscattering and meteorological visual range," Appl. Opt. 5, 293-295 (1966).

28. S. Twomey and H. B. Howell, "Relative merit of white and monochromatic light for the determination of visibility by backscattering measurements," Appl. Opt. 4, 501-506 (1965).

29. G. L. Kunz, "Probing of the atmosphere with lidar," AGARD Conf. Proc. 23, 1-11 (1992).

30. J. D. Klett, "Lidar calibration and extinction coefficients," Appl. Opt. 22, 514-515 (1983).

31. R. T. Brown, "A new lidar for meteorological application," J. Appl. Meteorol. 12, 698-708 (1973).

32. L. R. Bissonnette and D. L. Hutt, "Multiple-scattering aerosol lidar inversion method," Can. J. Phys. 71, 39-46 (1993).

33. J. A. Weinman, "Derivation of atmospheric extinction profiles and wind speed over the ocean from a satellite-borne lidar," Appl. Opt. 27, 3994-4001 (1988). 